\title{
Correction to: Problems and Solutions in Nuclear and Particle Physics
}

\section{Correction to:}

S. Petrera, Problems and Solutions

in Nuclear and Particle Physics, https://doi.org/10.1007/978-3-030-19773-5

The original version of the book frontmatter (Preface and Notes) were revised. The references lists had been placed at the end of Preface and Notes for clarity in understanding.

The original version of the frontmatter was revised: The correction is available at https://doi.org/10.1007/978-3-030-19773-5 